BULLETIN Bulletin hispanique

HISPANIQUE Université Michel de Montaigne Bordeaux

119-1 | 2017

Autorité et pouvoir dans le théâtre du Siècle d'Or

\title{
El Recueil des phrases adverbiales et autres locutions qui ont le moins de rapport entre les deux Langues Espagnole \& Françoise (1647) \\ de Antoine Oudin
}

Ana M. Carranza Torrejón

\section{OpenEdition}

\section{Journals}

\section{Edición electrónica}

URL: https://journals.openedition.org/bulletinhispanique/4990

DOI: 10.4000/bulletinhispanique.4990

ISBN: 979-10-300-0142-6

ISSN: 1775-3821

Editor

Presses universitaires de Bordeaux

Edición impresa

Fecha de publicación: 15 junio 2017

Paginación: 371-388

ISBN: 979-10-300-0141-9

ISSN: 0007-4640

Referencia electrónica

Ana M. Carranza Torrejón, «El Recueil des phrases adverbiales et autres locutions qui ont le moins de rapport entre les deux Langues Espagnole \& Françoise (1647)», Bulletin hispanique [En línea], 119-1 | 2017, Publicado el 17 junio 2020, consultado el 21 septiembre 2021. URL: http://journals.openedition.org/ bulletinhispanique/4990 ; DOI: https://doi.org/10.4000/bulletinhispanique.4990 


\title{
El Recueil des phrases adverbiales et autres locutions qui ont le moins de rapport entre les deux Langues Espagnole \& Françoise (1647), de Antoine Oudin
}

\author{
Ana M. Carranza Torrejón \\ Gines - España
}

Cet article cherche à mettre en relief la singularité du Recueil des phrases adverbiales et autres locutions (1647), un répertoire lexical mixte qui est peut-être l'ouvrage pour apprendre l'espagnol le moins connu d'Antoine Oudin.

Mots-clés: Antoine Oudin, lexicographie, nomenclature, enseignement de l'espagnol.

Este artículo ahonda en las características de la que quizá sea la obra para aprender español más desconocida de Antoine Oudin, el Recueil des phrases adverbiales et autres locutions (1647), para poner de manifiesto la singularidad de este repertorio léxico mixto.

Palabras clave: Antoine Oudin, lexicografía, nomenclatura, enseñanza del español.

This article delves into the characteristics of what may be the most unknown work to learn Spanish, by Antoine Oudin, "Recueil des phrases adverbiales et autres locutions" (1647), to assert the uniqueness of this mixed lexical repertoire.

Keywords: Antoine Oudin, lexicography, lexical repertoire whose material is categorized by topics, History of the Teaching of Spanish as a Foreign Language. 


\section{INTRODUCCIÓN}

Afamado gramático francés, Antoine Oudin (1595-1653) es autor de obras capitales en las que ofrece un temprano testimonio fidedigno de los cambios acaecidos en la lengua francesa por influencia del bon usage ${ }^{1}$. Es igualmente conocido por los libros en los que pone en paralelo el francés y el italiano, quizá el idioma extranjero que más estimaba y con el que sin duda debió familiarizarse durante sus estancias en distintas cortes italianas. Algunas de esas obras para aprender italiano tienen mucho que ver con las que destinó a la enseñanza del español, conjunto en el que se incluye el Recueil des phrases adverbiales et autres locutions, qui ont le moins de rapport entre les deux Langues Espagnole \& Françoise -en adelante, Recueil-, que, por lo que sabemos, no fue editado más que una vez en París, por Antoine Sommaville, en 1647. De este opúsculo en $12^{\circ}$ de poco más de cien páginas sólo conocemos el ejemplar anejado a la Nomenclature françoise et espagnole (1647) -en adelante, Nomenclature- que atesora la Bibliothèque nationale de France (X-14228 (1) y (2)), un repertorio temático que tampoco tuvo más ediciones, pero sí bastante repercusión entre las nomenclaturas para aprender español publicadas en Francia hasta principios del XVIII ${ }^{2}$.

1. Véase, por ejemplo, el estudio de Ferdinand Brunot, Histoire de la langue française des origines à nos jours. 3, La formation de la langue classique (1600-1660). París, Armand Colin, 1966, p. 28-30.

2. Cabe recordar aquí que el término nomenclatura, en francés nomenclature, no tenía en el XVII ni la acepción de "conjunto de voces técnicas propias de una ciencia» ni tampoco la de "conjunto de entradas de un diccionario». Como explica A. Furetière en la definición que da del término en su Dictionaire Vniversel (1694), nomenclature, era la denominación habitual para un «catalogue de plusieurs mots les plus ordinairez d'une Langue, pour en faciliter l'usage à ceux à qui on l'Enseigne. On a fait plusieurs Nomenclatures Italiennes, Espagnoles ou des Abregez de Dictionnaires». Cierto es que las nomenclaturas son repertorios más o menos breves, de marcado carácter didáctico, pero Furetière pasa por alto el rasgo que las distingue de manera más clara de otros repertorios léxicos: la ordenación temática del caudal. Sobre la caracterización de las nomenclaturas pueden consultarse los trabajos de Bernard Quemada, Les dictionnaires du français moderne 1539-1863: Étude sur leur histoire, leurs types et leurs méthodes, París, Didier, 1968, p. 361-368, o los trabajos de Manuel Alvar Ezquerra, «Apuntes para la historia de las nomenclaturas en español», en Rafael González Tirado (ed.), Actas del VII Congreso Internacional de Lingüistica y Filología de América Latina (ALFAL). Homenaje a Pedro Henríquez Ureña, Santo Domingo, Universidad Nacional Pedro Henríquez Ureña, t. I, p. 457-470, Marta C. Ayala Castro, «Nomenclatures de l'espagnol (1526-1800). Considérations générales sur la nature et la fonction des nomenclatures», Cahiers de lexicologie, no 61-2, 1992, p. 127-160, y «El concepto de nomenclatura», en M. Alvar Ezquerra (ed.), Actas del IV Congreso Internacional EURALEX'90, Barcelona, Bibliograf, 1992, p. 437-444, M. Ángeles García Aranda, Un capitulo de la lexicografía didáctica del español: nomenclaturas hispanolatinas (1493-1745), tesis doctoral de la Universidad Complutense, 2003, p. 48-74, y Ana M. Carranza Torrejón, El vocabulario de la indumentaria de los siglos XVI a XIX. Estudio contrastivo a partir de las nomenclaturas con el francés y el español, tesis doctoral de la Universidad de Sevilla, 2012, p. 140-201. En nuestra tesis dedicamos igualmente un apartado a la descripción de la nomenclatura de Antoine Oudin en el que mostramos la influencia de ese repertorio en otros posteriores, Ibid., p. 230-243. 
Si la Nomenclature ha sido objeto de análisis por parte de distintos investigadores, en su mayoría hispanistas ${ }^{3}$, el Recueil ha pasado prácticamente desapercibido, y esto a pesar de ser una obra bastante singular. Aunque el título no lo indica, este repertorio contiene dos colecciones bilingües francés-español de muy distinta naturaleza: la primera, el "Recueil de Phrases Aduerbiales: Auec l'vsage des Prepositions et Conjonctions» (p.3-81), es un listado alfabético compuesto fundamentalmente por conjunciones y preposiciones, y, la segunda, titulada «Verbes d'habitudes ordinaires et actions en general» (p. 82118), agrupa exclusivamente verbos distribuidos por temas. El Recueil presenta así una estructura inusual en la lexicografía francoespañola de la época. Cabe preguntarse qué empujó a Antoine Oudin a dar a la luz este repertorio léxico mixto, cuál es su origen y su función dentro del conjunto de obras para aprender español del XVII. Al igual que en la Nomenclature, no hay preliminares que indiquen las condiciones en las que el gramático francés compuso el Recueil o sus intenciones, de modo que, para empezar a responder a estas preguntas, se hace necesario conocer el contexto en el que se surge este repertorio y cómo se integra con el resto de libros para aprender español del propio Antoine Oudin.

\section{LOS INICIOS DE LA ENSEÑANZA DEL ESPAÑOL EN FRANCIA}

Como es sabido, la influencia de la lengua y la cultura españolas en Francia tuvo su época dorada en el periodo comprendido entre el enlace de Luis XIII con Ana de Austria y el de Luis XIV con María Teresa de Austria, es decir, entre 1615 y $1660^{4}$.

El español compartió entonces protagonismo con el italiano, una lengua extranjera cuya enseñanza estaba bastante arraigada en Francia. No en vano, lo hemos dicho, Antoine Oudin, más que su padre, le dedicó varios libros a ese idioma, además de ejercer como maestro de Luis XIV para quien también trabajaba como secretario intérprete real, cargo en el que sucedió a su progenitor. Con todo, la breve españolización de la corte francesa favoreció notablemente la multiplicación de la producción editorial de libros que, a tenor de lo apuntado por los autores en los preliminares, estaban llamados

3. Véanse B. Quemada, Ibid., p. 365-366, M. C. Ayala Castro, Ibid., p. 139, M. A. García Aranda, Ibid., p. 140-141, Lidio Nieto Jiménez y Manuel Alvar Ezquerra, Nuevo Tesoro Lexicográfico del Español (siglo XIV-1729), Madrid, Arco/Libros, 2007, p. LXVI, Juan Crespo Hidalgo y Esperanza Olarte Stampa, «Segunda parte del estudio crítico-comparativo del Tesoro de la lengua castellana abreviado con nomenclaturas de los siglos XVI al XVIII", Analecta Malacitana Electrónica, no 25, 2008, p. 53-79, Luis Pablo Núñez, El arte de las palabras: diccionarios e imprenta en el Siglo de Oro, Mérida, Editora Regional de Extremadura, 2010, p. 117-122.

4. Sobre la influencia del español en Francia en esa época pueden consultarse, entre otros, el estudio de Alejandro Cioranescu, Le masque et le visage. Du baroque espagnol au classicisme français, Ginebra, Droz, 1983, o la tesis doctoral de Marie-Hélène Maux-Piovano, Les débuts de la didactique de l'espagnol en France : les premières grammaires pratiques (1596-1660), Villeneuve-d'Ascq, Presses Universitaires de Septentrion, 2002. 
a satisfacer las necesidades y los gustos de un público bastante amplio que por distintas razones debía desenvolverse en español. En este contexto tan favorable se pusieron en circulación obras de carácter didáctico de muy diversa naturaleza y calidad: gramáticas, diccionarios, recopilaciones de carácter paremiológico, traducciones o libros que proponen amenas lecturas, a veces en versión bilingüe. En este amplio abanico tienen cabida, por tanto, obras destinadas a eruditos y otras a personas menos instruidas, algunos libros más aptos para iniciarse en el conocimiento del castellano y otros para consolidar y ampliar el dominio de esa lengua.

Buena muestra de esa variedad es la obra de César Oudin, cuyo núcleo está compuesto por las que durante mucho tiempo se han considerado herramientas básicas para el aprendizaje de una lengua extranjera, esto es, una gramática, la Grammaire et Observations de la langue Espagnolle (1597), un diccionario, el Tesoro de las dos lenguas francesa y española (1607), considerado un hito de la lexicografía francoespañola, y una colección de diálogos, los Dialogos muy apazibles (1608). En un principio este último libro reunía siete diálogos en versión bilingüe español-francés inspirados en la colección Pleasant and delightfull dialogues in Spanish and English (1599), de John Minsheu, pero, más adelante, en la edición de 1622, se amplió el contenido con un octavo diálogo tras el cual se ańadió un «Nomenclator o registro de algunas cosas curiosas y necessarias de saberse, a los estudiosos de la lengua española», también españolfrancés, cuyo origen reside en la nomenclatura incluida en el libro Advertencia y breve metodo para saber leer, escrivir y pronunciar la lengua castellana (1615), de Lorenzo de Robles. A todas estas obras se suman, además, una recopilación de Refranes o proverbios Españoles traduzidos en lengua Francesa (1605), ediciones en castellano o en versión bilingüe de diversos títulos de la literatura española del Siglo de Oro, y traducciones, entre las cuales destaca la de la primera parte de El Quijote (1614)5.

La vigencia de la obra didáctica de César Oudin durante prácticamente todo el siglo quedó asegurada gracias a la labor de revisión que acometió su hijo. El segundo Oudin se centró en los tres títulos imprescindibles, aunque con un resultado desigual. Sus aportaciones a la Grammaire espagnole, expliquée en françois (1645) no parecen haber sido especialmente relevantes ${ }^{6}$. Notable es, en cambio, su contribución al diccionario bilingüe (1645), y en particular a la parte francés-español ${ }^{7}$. Bastantes diferencias presenta igualmente su edición del libro

5. Hace poco, Javier Suso insistía en la idea de que la obra de C. Oudin ha de entenderse como un conjunto de recursos que ofrece un completo programa pedagógico para aprender castellano. Véase "La enseñanza del español en Francia en el siglo XVII», en Antonio Manuel González Carrillo (coord.), Post tenebras spero lucem. Los estudios gramaticales en la España medieval y renacentista, Granada, Editorial de la Universidad de Granada, p. 379-403.

6. Esa es la conclusión a la que llega Isolina Sánchez Regueira en su artículo «Antoine Oudin, gramático francés entre Maupas y Vaugelas», Verba, no 12, 1985, p. 361-382.

7. Sobre las diferencias que existen entre las ediciones de los dos Oudin puede consultarse otro artículo de Isolina Sánchez Regueira: "César et Antoine Oudin: étude comparative des éditions de son 'Thrésor' et son importance dans le domaine de la lexicographie», Verba, 9, 1985, p. 329-340. 
de diálogos (1650): en su revisión, el segundo Oudin convirtió esos diálogos en una colección tetralingüe, pues a la traducción francesa del texto español añadió la versión del texto en alemán y en italiano, prescindiendo, además, de la nomenclatura español-francés anejada en determinadas ediciones ${ }^{8}$. Parece lógico que el afamado gramático no se interesara por este repertorio, puesto que para entonces había publicado su ya citada Nomenclature (1647). Esta y el Recueil son las dos obras para aprender espańol más originales del segundo Oudin. Lo son porque, por una parte, no entroncan con ningún trabajo de su padre, sino con dos obras suyas para aprender italiano publicadas unos años antes: la Nomenclature françoise et italienne (1643) y el Petit recueil des phrases adverbiales et autres locutions, qui ont le moins de rapport entre les deux Langues Italienne \& Françoise (1646). Por otra parte, los dos repertorios que comentamos se desmarcan del conjunto de obras lexicográficas para aprender español publicadas en Francia hasta 1647.

En esa época el referente imprescindible en el terreno lexicográfico era el Tesoro, un amplio diccionario alfabético bidireccional de gran formato que, como tal, era considerado entonces un "ouvrage de bibliothèque»" , es decir, una obra al alcance de unos pocos privilegiados. Más asequibles eran las nomenclaturas, repertorios onomasiológicos monodireccionales que, por su brevedad, se acomodaban fácilmente como auxiliares dentro de obras mayores. Así se publicaron las cuatro nomenclaturas destinadas a la enseñanza del español en Francia anteriores a la de Antoine Oudin, todas bilingües españolfrancés: la ya citada de Lorenzo de Robles (1615), base del también mencionado «Nomenclator» incluido en los Diálogos (1622) de César Oudin, que está a su vez en el origen de la nomenclatura incluida en el Thesoro de diversa lición (1636), de Ambrosio de Salazar, y el breve «Diccionario por su orden» incluido al final del Ramilete de flores poeticas (1620) de Alejandro de Luna.

La Nomenclature de Antoine Oudin, en cambio, no forma parte de ninguna otra obra y se presenta con una combinación de lenguas inversa: el castellano no es lengua de entrada, sino de llegada. El repertorio de 1647 descuella igualmente por el amplio caudal registrado -6.852 entradas, frente a, por ejemplo, las $1.343 \mathrm{del}$ «Nomenclator» del primer Oudin-, distribuido en sesenta y cuatro capítulos temáticos. Dentro de estos, es habitual que las entradas aparezcan dispuestas bien alfabéticamente, bien con una distribución semántica, rasgo que marca una clara diferencia con respecto a las nomenclaturas anteriores, en las que el contenido de los apartados temáticos no suele responder a un orden aparente. En su nomenclatura, además, Antoine Oudin dio clara prioridad a las equivalencias unívocas, esto es, a aquellas en las que a una voz de entrada le corresponde otra en la lengua de llegada, evitando

8. En nuestra tesis (op. cit., p. 14-16) pueden encontrarse los detalles sobre las distintas ediciones de ese «Nomenclator».

9. Empleamos aquí la expresión usada por Laurent Bray en «Consultabilité et lisibilité du dictionnaire, aspects formels», en F.-J. Hausmann et al., Wörterbücher. Ein internationales Handbuch zur Lexikographie, Berlín/Nueva York, Walter de Gruyter, t. I, p. 135-146. 
por tanto la acumulación de voces en una y otra lengua, así como las perífrasis definitorias que pudieran hacer las veces de equivalente o aportar algún matiz a la traducción propuesta. Al igual que sus precedentes, la Nomenclature está compuesta mayoritariamente por sustantivos, aunque determinados capítulos cuentan con una sección exclusiva de adjetivos, categoría gramatical que no aparece más que de manera puntual tanto en las nomenclaturas anteriores como en muchas de las posteriores. Por último, cabe destacar que, si sus predecesores no fueron rigurosos a la hora de incluir información adicional, como el género y el número de los sustantivos registrados, indicado a través de un artículo determinado o indeterminado, Antoine Oudin fue bastante sistemático a la hora de excluir la información de carácter gramatical. La microestructura es precisamente uno de los aspectos que más acercan el Recueil a la Nomenclature. Cabe esperar que ambos repertorios compartan algún rasgo, más aún teniendo en cuenta que, como hemos seńalado, la segunda parte del Recueil es una recopilación temática, si bien en este caso compuesta exclusivamente por verbos. Con todo, tanto esta como la primera colección -que, recordemos, es alfabética- tienen características particulares que describimos a continuación.

\section{EL RECUEIL DES PHRASES ADVERBIALES ET AUTRES LOCUTIONS}

\section{El «Recueil des phrases adverbiales: Auec l'vsage des Prepositions et Conjonctions»}

La colección anunciada en el título encaja, al menos en parte, en el patrón de otra obra a la que Antoine Oudin se refería en la introducción de sus Recherches italiennes et françoises (1640), cuando apuntaba que se había dedicado a "recueillir les phrases Italiennes les plus difíciles à entendre, auec dessein d'en faire vn corps de liure à part [...]» ${ }^{10}$. Este proyecto es el que parece haber desembocado en el Petit recueil des phrases adverbiales (1646) francés-italiano, la base que posteriormente adaptó el propio Oudin con el fin de recopilar sintagmas y expresiones que, tal y como reza en el título del Recueil de 1647, "ont le moins de rapport entre les deux Langues Espagnole \& Françoise». El grueso de las entradas lo componen preposiciones y conjunciones que se repiten en distintos sintagmas, en su mayoría seleccionados desde una perspectiva contrastiva francés-español:

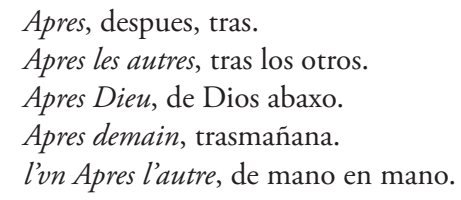

10. Antoine Oudin, Recherches italiennes et françoises, París, Antoine de Sommaville, 1640, p. 27. 
l'Apresdisnée, despues de comer, el tiempo despues de la comida.

Apres moy, despues de mi. (1647, p. 6)

Aussi, tambien, aun.

Aussi, y por esto.

Aussi faut-il, pues es menester.

Aussi que, de mas que.

Aussi veux-ie, assi quiero yo.

Aussi bien, ni mas ni menos.

Aussi bien l'un que l'autre, assi el vno como el otro.

et moy Aussi, yo tambien.

Aussi peu, tampoco.

Aussi tost, luego luego.

Aussi-tost fait aussi-tost dit, dicho y hecho.

Aussi-tost que, luego que.

Aussi long-temps, tanto tiempo, quanto tiempo (1647, p. 9).

En esta recopilación de poco más de 1.600 entradas también tienen cabida adverbios que, como «courageusement, esforçadamente», pueden quedar registrados inmediatamente después del sustantivo del que derivan -en este caso, «courage, animo» (1647, p. 19) - o tras alguno de los contados adjetivos, consignados por lo general en expresiones del tipo «à Belles dents, a poder de dientes", "de plus Belle, de nueuo", entradas seguidas de "Bellement, gentiment, lindamente» (1647, p. 10). No obstante, los pocos adjetivos incluidos en esta primera recopilación no suelen dar pie más que a una entrada, como "à l'espée Blanche, con la espada, con espadas desuaynadas» (1647, p. 11) o «habit tout Vny, vestido llano» (1647, p. 80). No ocurre lo mismo con los sustantivos que también se intercalan en este primer listado y que, al igual que la gran mayoría de preposiciones, conjunciones o adverbios, dan lugar a pequeñas series, como las que reproducimos a continuación:

venir à Bout, salir con vna cosa.

sur le Bout du doigt, de coro, de corrida.

à chaque Bout de champ, a cada trecho.

à Bout portant, con la punta del pistolete sobre el pecho.

de Bout en bout, de vn cabo al otro (1647, p. 12).

à la Mode, al vso.

à ma Mode, a mi fantasia, a mi modo.

fait à la Mode, par ironie, hecho a priessa y mal (1647, p. 47).

de plein Gré, de muy buena gana.

à Gré, a gusto, a pelo.

à mon Gré, a mi gusto.

de son bon Gré, de buena gana.

bon Gré mal gré, por amor o por fuerça.

contre son Gré, contra su voluntad.

sçauoir bon Gré, agradescer.

sçauoir maunais Gré, desgraciar (1647, p. 37). 
En estos ejemplos se puede constatar que las entradas se repiten en expresiones pluriverbales de diferente catalogación: las hay tanto lexicalizadas - «sur le Bout du doigt» o "bon Gré mal gré»- como no lexicalizadas - Apres moy» $\mathrm{o}$ «et moy Aussi»-, aunque, salvo alguna excepción, contienen una palabra previamente lematizada. Los ejemplos muestran igualmente que, de manera ocasional, Antoine Oudin añade una segunda traducción en español aportando así una palabra o expresión sinónima, como «despues de comer, el tiempo despues de la comida» $\mathrm{o}$ "tanto tiempo, quanto tiempo». No hay definiciones, pero las distintas series que se forman vienen a ilustrar el contexto en el que se puede emplear cada entrada y su equivalente.

Se trata de un rasgo particular de esta primera recopilación, pues no hay ejemplos del uso pragmático del vocabulario recogido en la segunda recopilación, compuesta fundamentalmente por verbos registrados en equivalencias unívocas. Cierto es que en ocasiones se hace necesario añadir alguna aclaración acerca del vocabulario que se está enseñando, más aún cuando la intención es dar a conocer el empleo de expresiones que difieren notablemente al poner en paralelo dos lenguas. De manera puntual, en la primera colección del Recueil pueden encontrarse marcas, abreviadas o no, pero siempre en francés, para señalar el uso particular de ciertas entradas, como en el extracto anterior "Fait à la Mode, par ironie, hecho a priessa y mal» o en «à son Corps deffendant, metaph., contra su voluntad» (1647, p. 18; el resaltado, en estos ejemplos y en los siguientes, es nuestro). Otras veces no es una marca, sino otra expresión afín a la entrada francesa la que aclara el sentido figurado o metafórico de la correspondencia propuesta, como en «se couper la Gorge, se battre, matarse» (1647, p. 36) o «il est Grec, il est habile, le Castillan dit, es latino» (1647, p. 37). En este último ejemplo y en los dos siguientes, Antoine Oudin incluye una acotación para precisar el contexto exacto en el que ha de emplearse la expresión registrada: «il est fait comme de Cire, qui se dit d’vn habit, entalla bien, viene de molde» (1647, p. 16) y «Vie vie, adelante, ea camina. Cela se dit en chassant vne personne» (1647, p. 79).

A pesar de resultar especialmente práctico en lexicografía bilingüe, este tipo de añadidos resulta inhabitual en los repertorios franceses de carácter didáctico de la época: de hecho, no los encontramos en la recopilación de verbos del Recueil ${ }^{11}$. Con todo, hemos de destacar esta característica del primer repertorio de Antoine Oudin, entre otras razones porque marca una diferencia con respecto al correspondiente de la versión francés-italiano del Recueil (1646). Hemos dicho que la columna del francés de esta otra recopilación es la base fundamental de la que analizamos. Sin embargo, en 1646 apenas si hay añadidos de cualquier clase: los casos citados hasta ahora, por ejemplo, no figuran en ese listado. Aunque no idénticas, esas indicaciones aparecen en la otra fuente que debió resultar particularmente útil a Antoine Oudin para componer sus repertorios francés-español: el Tesoro de las dos lenguas. En la

11. Aunque son pocas, en la Nomenclature sí se incluyen glosas, que vienen ya a suplir la falta de equivalente en español, ya a aclarar el significado de la voz castellana. 
parte francés-español de la edición revisada por el segundo Oudin encontramos consignadas las que muy probablemente sean las entradas que dieron lugar a las incorporaciones en la versión del Recueil de 1647 a las que acabamos de aludir:

Fait à la Mode, hecho a priessa.

À son Corps deffendant. i. contra su voluntad.

Se couper la Gorge, matarse.

il est Grec. i. sabe mucho, es Latino.

Ciré fait comme de Cire, qui se dit d'vn habit, etc. que viene de molde, que entalla bien.

Vie, vie, qui se dit pour chasser quelqu'un, ea camina, adelante.

À l'espee Blanche, con la espada desvaynada [sic] (Tesoro, 1645).

La primera colección del Recueil contiene, por tanto, una selección de entradas previamente registradas en el Tesoro que se suman a las extraídas de la versión francés-italiano del «Recueil des phrases adverbiales», de modo que el listado de 1647 cuenta también con entradas inéditas en el diccionario, tal y como puede comprobarse en la siguiente tabla:

\begin{tabular}{|l|l|l|}
\hline \multicolumn{1}{|c|}{ Tesoro, $\mathbf{1 6 4 5}$} & \multicolumn{1}{|c|}{ Recueil, $\mathbf{1 6 4 6}$} & \multicolumn{1}{c|}{ Recueil, $\mathbf{1 6 4 7}$} \\
\hline Mode, modo de verbo. & - & - \\
\hline Mode, modo, manera, vso. & - & - \\
\hline à la Mode, al vso. & $\begin{array}{l}\text { à la Mode, all'usanza, alla } \\
\text { foggia. }\end{array}$ & à la Mode, al vso. \\
\hline - & $\begin{array}{l}\text { à ma Mode, à mia posta, } \\
\text { à mia voglia, à mio modo } \\
(1646, \text { p. 38) }\end{array}$ & $\begin{array}{l}\text { à ma Mode, a mi fantasia, a } \\
\text { mi modo. }\end{array}$ \\
\hline $\begin{array}{l}\mid \\
\text { fait à la Mode, hecho a pries- } \\
\text { sa. }\end{array}$ & $\begin{array}{l}\text { fait à la Mode, par ironie, } \\
\text { hecho a priessa y mal (1647, } \\
\text { p. 47). }\end{array}$ \\
\hline
\end{tabular}

\section{«Verbes d'habitudes ordinaires et actions en general»}

La conexión con el Tesoro no resulta tan evidente en la que es la primera y única recopilación onomasiológica independiente de verbos con el francés y el español que hemos encontrado. El título pone de manifiesto que la segunda parte del Recueil reúne una selección de verbos hecha no ya aplicando un criterio contrastivo, sino de frecuencia de uso. Esta diferencia explica el parecido con la correspondiente versión francés-italiano, evidente al cotejar la primera sección temática ${ }^{12}$ :

12. En el original de 1646 , las entradas en francés se presentan en redonda y las equivalencias en italiano, en cursiva. Hemos intercambiado el tipo de fuente para adaptar el extracto al formato que presenta el original de 1647. 


\begin{tabular}{|l|l|}
\hline \multicolumn{1}{|c|}{ Recueil, $\mathbf{1 6 4 6}$} & \multicolumn{1}{c|}{ Recueil, $\mathbf{1 6 4 7}$} \\
\hline S'éueiller, suegliarsi. & S'Esueiller, despertar. \\
\hline \hline- & Esueiller vn autre, recordar despertar a otro. \\
\hline Se leuer, leuarsi. & Se leuer, leuantarse. \\
\hline Se chausser, calzarsi. & Se chausser, calçarse. \\
\hline Se peigner, pettinarsi. & Se peigner, peynarse. \\
\hline Se curer les oreilles, stuzzicarsi le orecchie. & Se curer les oreilles, escaruarse las orejas. \\
\hline S'habiller, vestirsi. & S'habiller, vestirse. \\
\hline \hline- & Prendre la botte, calçar botas. \\
\hline Se coiffer, acconciarsi il capo. & Se coiffer, tocarse, atauiarse. \\
\hline S'ajuster, s'agencer, acconciarsi. & S'ajuster, adereçarse. \\
\hline Se parer, ornarsi. & Se parer, ornarse. \\
\hline Se farder, imbellettarsi. & Se farder, afeytarse. \\
\hline S'attacher, attacarsi. & S'attacher, atarse. \\
\hline Se boutonner, abbottonarsi. & Se boutonner, botonarse, abrocharse. \\
\hline Se lacer, allaciarsi. & Se lacer, enlazarse, abrocharse. \\
\hline Noüer, annodare. & Noüer, anudar. \\
\hline- & Lier, atar, ligar. \\
\hline- & Serrer, estreindre, estreñir. \\
\hline S'aggraffer, vncinarsi. & S'aggraffer, heuillarse. \\
\hline \hline
\end{tabular}

La columna del francés de 1646 está, salvo por la voz francesa «s'agencer», recogida en su totalidad en la correspondiente de 1647. Ahora bien, en esta otra lista se intercalan nuevas entradas con verbos semánticamente afines a los ya registrados, de manera que las incorporaciones no interrumpen el orden interno que rige esta colección, compuesta por 836 entradas distribuidas en veintiséis secciones temáticas separadas por una raya.

Por tanto, además de por el criterio aplicado para seleccionar el vocabulario consignado, las dos recopilaciones que componen el Receuil se distinguen netamente por la ordenación del caudal. El orden alfabético de la primera se explica porque registra fundamentalmente conjunciones y preposiciones, elementos gramaticales, no referenciales, los cuales no se prestan a una ordenación por temas como ocurre con los sustantivos y adjetivos que componen la Nomenclature y con los verbos concentrados en la segunda parte del Recueil, dos repertorios que presentan bastantes paralelismos a nivel de organización, cuestión en la que profundizamos más adelante. Ahora estimamos más oportuno insistir en lo que implica ordenar el léxico según un criterio semántico, es decir, atendiendo al sentido de las voces registradas, y no a su forma, o, en términos del siglo XVII, clasificar el caudal «por su orden» o por el "orden natural», y no "por el A, B, C». A este último se le atribuía ya entonces la gran ventaja de facilitar la localización de las voces, mientras que el primero se consideraba mucho más provechoso para el aprendizaje memorístico del vocabulario. Al menos así lo han puesto de manifiesto diversos autores más o menos coetáneos de Antoine Oudin. Por ejemplo, el mismo año que éste publicó la Nomenclature y el Recueil, Claude Favre de Vaugelas dio a la luz 
sus célebres Remarques sur la langue françoise (1647), en cuyo texto preliminar argumentaba del siguiente modo su opinión acerca del ordenamiento alfabético del caudal:

Outre que cét [sic] ordre Alphabetique ne produit de soy autre chose, que de faire trouuer les matieres plus promptement; c'est pourquoy il a tousiours esté estimé le dernier de tous les ordres, qui ne contribuë rien à l'inteligence des matieres que l'on traite; Et de fait pour en donner vn exemple tout visible, entendroit-on mieux la remarque que ie fais sur ce mot amour, et celle que ie fais sur la preposition auec, sils estoient tous deux rangez sous vne mesme lettre? Ont-ils quelque chose de commun ensemble, si ce n'est de commencer par vne mesme lettre, qui n'est rien? ?3 $^{13}$

Tampoco era partidario Vaugelas de la clasificación según «vne autre espece d'ordre à garder plus raisonnable et plus vtile, qui est de ranger toutes ces Remarques sous les neuf parties de l'Oraison», pues, a su parecer, "il ne seruiroit qu'à ceux qui sçauent la langue latine, et par consequent toutes les parties de la Grammaire» ${ }^{14}$. Por lo que hemos explicado hasta ahora sobre el Recueil y la Nomenclature, Antoine Oudin sí optó por la clasificación de las voces según su categoría gramatical, alejándose así de los repertorios para aprender vocabulario español -casi todos temáticos- publicados en Francia antes que los suyos. En esos otros, esta distribución no ha lugar porque la presencia de verbos y adjetivos es puntual y la de adverbios, preposiciones y conjunciones, nula. Cierto es que, como decía Vaugelas, la clasificación por categorías gramaticales podía resultar más ventajosa para personas instruidas, que no son necesariamente el público al que se dirigen las nomenclaturas del XVII. Sin embargo, parece que Antoine Oudin decidió comenzar estableciendo esa división con intención de dar al caudal el tratamiento que estimó más oportuno de cada vez para obtener como resultado un repertorio de eminente carácter didáctico. De esta forma, siempre que la naturaleza de las voces así se lo permitiera, Antoine Oudin recurrió al orden temático, que, lo hemos apuntado, se ha considerado tradicionalmente mucho más propicio para el aprendizaje. Esto es lo que recogen testimonios como el de Francisco Sobrino, célebre maestro de español y gran conocedor de la obra de los dos Oudin, quien justificaba la presencia de una nomenclatura francés-español al final de la primera edición de su Diccionario Nuevo (1705) con las siguientes palabras ${ }^{15}$ :

13. Claude Favre de Vaugelas, Remarques sur la langue françoise vtiles a ceux qui veulent bien parler et bien escrire, París, Vda. Jean Camusat y Pierre Le Petit, 1647, «Preface», p. s. n.

14. C. F. de Vaugelas, Ibid., p. s. n.

15. En el texto que reproducimos a continuación, Sobrino remoza en buena medida el prefacio del Indiculus vniversalis (1667), de François Pomey, nomenclatura francés-latín que adaptó el maestro de español y que publicó bajo el título de "Methode facile pour aprendre la langue espagnole; par l'arangement des mots selon la construction de l'Univers, avec les principaux termes des Arts \& des Sciences» (1705). En lugar de aludir al orden natural o de las cosas, Pomey alababa las ventajas del diálogo como herramienta didáctica al describirlo como la «manière la plus methodique et la propre à insinuer et faire comprendre les choses». Justificaba así la presencia de textos dialogados en su nomenclatura, en particular en la primera parte. 
Comme la plus grande difficulté qui rebute au commencement les Ecoliers d'une Langue, est de trouver et d'aprendre les termes les plus communs, et bien souvent les plus ignorés de ceux qui y sont les plus avancés; on a cru, pour aplanir ces difficultés, devoir joindre à ce Dictionaire François-Espagnol un Recueil abregé, ou un Précis de tous les termes de l'une et l'autre Langue, disposés selon l'ordre des choses, comme ils sont disposés dans le Dictionaire selon l'ordre des lettres; affin que l'esprit les regardant dans leur ordre naturel, on puisse plus facilement les concevoir, et retenir les termes qui les representent ${ }^{16}$.

El testimonio de Sobrino resulta contundente: la ordenación del léxico en función del sentido de las voces registradas y no de su forma facilita la comprensión del significado de las mismas y su memorización, mientras que un diccionario bilingüe alfabético, como el suyo o como el Tesoro, no se presta al aprendizaje sistemático del vocabulario de una lengua extranjera. No obstante, lo hemos señalado, Antoine Oudin no renunció por completo al orden alfabético ni en el Recueil ni en la Nomenclature, si bien el criterio formal no es el que se aplica en primer lugar: en el Recueil, la clasificación inicial se establece según la naturaleza de las voces registradas, lo que da lugar a dos recopilaciones distintas; en la Nomenclature la ordenación alfabética de las entradas se hace dentro de un buen número de apartados temáticos, combinando de esta forma las ventajas de ambas clasificaciones.

Por lo general, la disposición de las voces «por su orden» se manifiesta a través de los epígrafes que establecen los grupos temáticos. Sin embargo, en la colección de verbos, a diferencia de lo que ocurre en la Nomenclature, no siempre hay un título que anuncie los temas abordados en cada sección, aunque el encabezado suele resultar bastante ilustrativo. Apuntamos a continuación la primera entrada y la última de las veintiséis secciones que componen esta segunda parte del Recueil, junto al número total de entradas en cada una (cifra entre paréntesis):

S'esueiller, despertar / S'agraffer, heuillarse (19)

Se deshabiller, desnudarse / Songer, soñar (20)

Faire la chambre, adereçar el aposento / Salir, ensuciar (11)

Aller ouyr la Messe, yr a oyr Missa / Communier, comulgar (6)

Mettre le counert, assentar la mesa / Oster le counert, desseruir, alçar la mesa,

quitar los manteles (40)

Allumer, encender / Vuider, vaziar (57)

Bailler, bostezar / Creuer, rebentar $(44)^{17}$

Voir, ver / Faire la mine ou maunais visage, despegarse (20)

Ouyr, oyr / Deuenir sourd, ensordecer (6)

Toucher, tocar / Sentir, sentir (15)

Flairer, oler / Sentir maunais, oler mal (3)

Aller, yr / Emmener, conduzir, lleuar (95)

16. Francisco Sobrino, Diccionario nuevo de las lenguas española y francesa, Bruselas, Francisco Foppens, 1705, p. 351.

17. Esta sección reúne fundamentalmente verbos relacionados con el sentido del gusto, además de verbos del tipo besar, bostezar, hablar, cantar, enmudecer, etc. Directamente relacionada con esa sección, las siguientes refieren verbos relacionados con los otros cuatro sentidos, tal y como puede comprobarse en el listado. 
Naistre, nascer / Mourir, morir (23)

Aimer, amar, querer / Mettre en manuaise humeur, amohinar (24)

Regner, reynar / Enuoyer, embiar (47)

Negocier, negociar / Eslargir, libertar, soltar de la carcel (26)

Attaquer, assaillir, acometer / Fuyr, huir (25)

Monter à Cheual, caualgar / Desteler, desuñir (20)

Leuer boutique, poner tienda, abrir / Seurer, destetar (56)

Saigner, sacar sangre / Prendre Medecine, tomar melezina (16)

Iouër d'vn instrument, tañer / Ramoner la cheminée, deshollinar (107)

Labourer, labrar la tierra, arar / Faire pastre, apascentar (29)

Ioüer, jugar / Enterrer, soterrar, sepultar (36)

Des Animaux, De los Animales (24)

Des choses inanimées, De las cosas inanimadas $(67)^{18}$

No deja de ser llamativo que tan solo las dos últimas secciones vayan encabezadas con un epígrafe: Antoine Oudin parece querer distinguir de forma clara los grupos de verbos que no guardan relación directa con las acciones propias del ser humano referidas en las secciones anteriores. Esta diferenciación pone de manifiesto la ordenación antropocéntrica de los temas de esta colección; en la Nomenclature, en cambio, los sesenta y cuatro capítulos temáticos establecidos por Antoine Oudin están dispuestos de acuerdo con el orden teocéntrico, es decir, el repertorio comienza con el epígrafe dedicado a «Dios», siguiendo con el universo y la naturaleza hasta el hombre, descrito tanto en el plano físico como en el ético y moral. A pesar de la distinta ordenación, existe cierta correspondencia entre los temas ilustrados en la colección de verbos y en la Nomenclature. En este otro repertorio hay, por ejemplo, varios apartados destinados a detallar la sociedad civil, terreno que abarca los diferentes oficios de hombres y mujeres; prácticamente todas esas profesiones están representadas en diferentes secciones de verbos mediante el registro de entradas que indican acciones propias de cada gremio, entre otros, médicos, músicos, pintores y escultores, libreros e impresores, mercaderes e incluso hay correspondencia con la sección de la Nomenclature dedicada a las "condiciones infames» (asesinos, truhanes, tahúres, salteadores...) en la serie de acciones recogidas tras los verbos relacionados con el juego, tema igualmente abordado en el último capítulo de la nomenclatura de sustantivos y adjetivos.

La conexión entre la segunda colección del Recueil y la Nomenclature es evidente no sólo a nivel de contenido sino también de forma. Apuntábamos antes que las entradas de 1647 que no aparecen en la versión de la colección de verbos francés-italiano se incorporan de acuerdo con el orden que rige la distribución del contenido dentro de los apartados temáticos. Ese orden interno puede verse claramente en el extracto correspondiente a la primera sección. Ahí se agrupan las acciones habituales del hombre al comenzar el día, esto es,

18. Este último apartado cuenta con dos secciones, la primera (43 entradas) empieza con «S'enraciner, prendre racine, arraygarse, tomar rays» y termina con «Sonner, sonar», y la segunda (24 entradas), relacionada con los fenómenos metereológicos, comienza con «Poindre, paroistre, assomar» y concluye con «Fumer, humear, echar humo». 
verbos afines a «'esueiller, despertar» y «s'habiller, vestirse». La siguiente sección recoge las acciones contrarias a estas, pero en sentido inverso, es decir, empieza por «se deshabiller, desnudarse» y termina con «songer, soñar», entrada cuyo contenido está semánticamente emparentado con «se coucher, acostarse». El resultado es un listado en el que las entradas se agrupan por afinidad semántica de acuerdo con el orden lógico en que se realizan las acciones designadas por los verbos.

Este tipo de disposición ordenada del contenido dentro de los propios apartados temáticos existe también en la Nomenclature. Por ejemplo, la enumeración de "Dignidades y profesiones temporales» (p. 122-126) sigue un orden jerárquico, de modo que el listado refleja la pirámide social, comenzando por el «Monarque, Monarca; Empereur, Emperador; Imperatrice, Emperiere, Emperadriz» y terminando por el «Prisonnier, Prisionero, Preso, encarcelado». Lo mismo ocurre con el capítulo dedicado a las «Dignidades espirituales» (p. 118-121), cuyo listado va encabezado por el «Pape, Pontife, Papa, Pontifice» y de ahí hasta la parte más baja de la pirámide, representada, entre otros, por el «Fossoyeur, Ahoyador, sepulturero» o los «Plereurs d'enterremens, Enlutados». Esta distribución ordenada del contenido, muestra de lo compleja que puede llegar a ser la macroestructura de los repertorios temáticos o nomenclaturas, es un aspecto particularmente relevante del trabajo de Antoine Oudin: no solo manifiesta su pericia como lexicógrafo, sino que también marca una clara diferencia con las nomenclaturas para aprender español publicadas en Francia antes de 1647. En estas, así como en bastantes nomenclaturas posteriores, el ordenamiento del caudal se suele limitar a la división del mismo en grupos temáticos, sin prestar mayor atención al orden interno en que se pueda disponer el contenido en cada uno de ellos con el fin de contribuir a facilitar el trabajo del aprendiz de lenguas extranjeras. Ahora bien, no sabemos si los lectores del Recueil supieron aprovechar los beneficios de la disposición de los verbos siguiendo un criterio semántico, a veces combinado con el orden lógico al que se prestan ciertos temas, y otras, únicamente agrupando las entradas por afinidad, jugando con las relaciones de sinonimia y antonimia. En cualquier caso, el resultado es una recopilación en la que el caudal presenta una organización interna inusual en la época, apuntando, además, algunas de las características del sistema ideológico que tanto juego ha dado en lexicografía sobre todo a partir del XIX.

Finalmente, debemos insistir en lo novedoso que resulta el hecho de que Antoine Oudin decidiera componer un repertorio temático exclusivamente de verbos. Ya hemos dicho que esta categoría gramatical aparece de forma puntual en unas pocas nomenclaturas para aprender español anteriores a 1647; entre las posteriores a esa fecha, en cambio, sí las hay que presentan una división por categorías gramaticales anterior a la distribución por temas. De hecho, en trabajos destacados sobre las nomenclaturas del español se señala la «Nomenclatura Hispanica Gallica» (p. 245-352), incluida en la Gramática \& sintaxis lingue Hispanice (1711), del maestro alemán Matthias Kramer, como 
la primera en contar con un apartado temático de verbos ${ }^{19}$. Quizá porque no lo conocían, las autoras de esas investigaciones no mencionan el Recueil de Antoine Oudin, que, según lo explicado, es un claro precedente de las nomenclaturas cuya estructura presenta una división por categorías gramaticales.

\section{Conclusión}

Hemos querido poner de manifiesto la singularidad de una de las obras menos conocidas de Antoine Oudin. Por su forma y contenido, el Recueil resulta ser un repertorio original, claramente alejado del resto de libros para aprender español publicados en Francia hasta mediados del siglo XVII. El hecho de que su origen resida en la obra del mismo Antoine Oudin ya puede entenderse como una novedad, pues establece una conexión entre distintos títulos que no existe ni siquiera en el conjunto de libros de su padre. El Recueil es un ejemplo bastante ilustrativo de cómo el segundo Oudin supo sacar partido a su propio trabajo, tanto por el hecho de haber tomado como base una obra anterior suya destinada a la enseñanza del italiano como por haber aprovechado su labor de revisión del Tesoro, una fuente de gran utilidad a la hora de adaptar el Recueil a la enseñanza del español. La conexión entre las distintas obras confiere una notable uniformidad al trabajo de Antoine Oudin. Esta asoma simplemente comprobando la presentación de las entradas: por ejemplo, una de las reformas importantes que acometió en el Tesoro consistió en evitar que una entrada fuera acompañada de dos o más equivalentes, un tipo de artículo lexicográfico que tiene una presencia limitada tanto en el Recueil como en la Nomenclature. La homogeneidad alcanza también al contenido: a lo largo de nuestra investigación hemos tenido ocasión de comprobar cómo en las obras que llevan su nombre una misma voz suele presentar las mismas equivalencias, algo que no siempre ocurre en la obra del primer Oudin.

Los rasgos que caracterizan al Recueil lo convierten, a nuestro entender, en un complemento perfecto de la Nomenclature, con la que forma un conjunto que, por sus dimensiones y su contenido, bien podría servir de diccionario "de faltriquera» o, retomando las palabras de A. Furetière, de «abregé de dictionnaire». Por su carácter unidireccional francés-español, su macroestructura y su formato reducido y económico, ni el Recueil y ni la Nomenclature - por separado o en conjunto- competían con otros repertorios alfabéticos o temáticos que pudieran circular en el mercado, incluidas la edición del Tesoro a cargo del propio Antoine Oudin y la nomenclatura anejada a los Dialogos de su padre.

Por último, no podemos dejar de señalar que, a pesar de su brevedad, el Recueil, tiene el interés particular de presentarse como un antecedente de repertorios posteriores y esto es en buena medida gracias a la aplicación del

19. Véanse los trabajos de Marta C. Ayala Castro, op. cit., 1992a, p. 145-146, y M. Ángeles García Aranda, op. cit., 2003, p. 668-670. 
criterio que permite distribuir el caudal por categorías gramaticales. A partir de esa división, Antoine Oudin compuso un repertorio alfabético, orden práctico para las categorías no referenciales como las conjunciones y las preposiciones, y otro temático, en el que además se hacen intervenir otros criterios lingüísticos a la hora de distribuir las entradas dentro de los propios apartados y cuyo resultado tiene importantes ventajas a nivel didáctico. El modelo que ofrece el trabajo de Antoine Oudin es muy similar al que encontramos sobre todo en repertorios publicados a partir del XIX. A modo de ejemplo, podemos citar el Diccionario manual de voces necesarias para el trato comun en las cinco lenguas española, italiana, francesa, inglesa y latina (1804), de Josef de Frutos, un repertorio léxico mixto compuesto por una nomenclatura de sustantivos y seis vocabularios -verbos, adjetivos, pronombres, preposiciones, conjunciones e interjecciones-, de los cuales dos, el de verbos y el de adjetivos, son alfabéticos.

\section{Bibliografía}

\section{Fuentes primarias}

Frutos Josef de, Diccionario manual de voces necesarias para el trato comun en las cinco lenguas española, italiana, francesa, inglesa y latina, para facilitar el uso de ellas a los que aprenden, Madrid, Gómez Fuentenebro y Compañía, 1804, 185 p.

Furetière Antoine, Dictionnaire vniversel, La Haya / Rótterdam, Arnout y Reinier Leers, 1690.

Luna Alejandro de, Ramilete de flores poeticas, y notables hieroglificos, en alabanza de las hermosas damas deste tiempo, Toulouse, Jean Maffre, 1620, 144 p.

Oudin Antoine, Recherches italiennes et françoises, París, Antoine de Sommaville, 1640, VIII-952 p.

- Nomenclature Françoise et Italienne ou les noms appellatifs de toutes les choses, París, Antoine de Sommaville, 1643, 440 p.

- Petit recueil des phrases adverbiales et autres locutions, qui ont le moins de rapport entre les deux langues italienne \& françoise, París, Antoine de Sommaville, 1646, 95 p.

- Nomenclature françoise et espagnole, París, Antoine de Sommaville, 1647, VII$438 \mathrm{p}$.

- Recueil des phrases aduerbiales et autres locutions, qui ont le moins de rapport entre les deux langues espagnole \& françoise, París, Antoine de Sommaville, 1647, 119 p.

- Dialogues fort recreatifs composez en espagnol, et nouvellement mis en italien, alleman, \& françois, París, Antoine de Sommaville, 1650, 544 p.

Oudin César, Dialogos muy apazibles, escritos en lengua española, y traduzidos en frances, París, Marc Orry, 1608, 167 p. Otra edición citada: París, Pierre Billaine, 1622, VI-192-40 p.

- Tesoro de las dos lenguas francesa y española, París, Marc Orry, 1607. Otra edición citada: Le Tresor des deux langues espagnolle et françoise de Cesar Oudin. [...] Le tout corrige \& reduit en meilleur ordre, par Antoine Oudin, París, Antoine de Sommaville, Augustin Courbé y Nicolas y Jean de la Coste, 1645.

Pomey François, Indiculus vniversalis, Lyon, Antoine Molin, 1667, 276 p.

Robles Lorenzo de, Advertencia y breve metodo para saber leer, escrivir y pronunciar la 
lengua castellana, París, Fleury Bourriquant, 1615.

Salazar Ambrosio de, Thesoro de diversa licion, obra digna de ser vista por su gran curiosidad, París, Louis Boullanger, 1636, 278 p.

Sobrino Francisco, Diccionario nuevo de las lenguas española y francesa, Bruselas, Francisco Foppens, 1705, 376 p.

Vaugelas Claude Favre de, Remarques sur la langue françoise vtiles a ceux qui veulent bien parler et bien escrire, París, Vda. Jean Camusat y Pierre Le Petit, 1647, 699 p.

\section{Estudios críticos}

Alvar Ezquerra Manuel, «Apuntes para la historia de las nomenclaturas en español», en González Tirado Rafael (ed.), Actas del VII Congreso Internacional de Lingüistica y Filología de América Latina (ALFAL). Homenaje a Pedro Henríquez Ureña, Santo Domingo, Universidad Nacional Pedro Henríquez Ureña, t. I, 1987, 590 p. También publicado en Alvar Ezquerra, Manuel, Lexicografía descriptiva, Barcelona, Bibliograf, 377 p.

Ayala Castro Marta C., «Nomenclatures de l'espagnol (1526-1800). Considérations générales sur la nature et la fonction des nomenclatures», Cahiers de lexicologie, 1992a, 2, no 61, 230 p.

Ayala Castro Marta C., «El concepto de nomenclatura», en Alvar Ezquerra Manuel (ed.), Actas del IV Congreso Internacional EURALEX'90, Barcelona, Bibliograf, 1992b, $532 \mathrm{p}$.

Bray Laurent, "Consultabilité et lisibilité du dictionnaire, aspects formels», en Hausmann Franz-Josef et al., Wörterbücher. Ein internationales Handbuch zur Lexikographie / Dictionnaires. Encyclopédie internationale de lexicographie, Berlín/Nueva York, Walter de Gruyter, 1989, 3 vols., 3355 p.

Brunot Ferdinand, Histoire de la langue française des origines à nos jours. 3, La formation de la langue classique (1600-1660). París, Armand Colin, 1966, 589 p.

Carranza Torrejón Ana M., El vocabulario de la indumentaria de los siglos XVI a XIX. Estudio contrastivo a partir de las nomenclaturas con el francés y el español, tesis doctoral de la Universidad de Sevilla, 2012, 806 p.

Cioranescu Alexandre, Le masque et le visage. Du baroque espagnol au classicisme français, Ginebra, Droz, 1983, 611 p.

Crespo Hidalgo Juan y Olarte Stampa Esperanza, «Segunda parte del estudio crítico-comparativo del Tesoro de la lengua castellana abreviado con nomenclaturas de los siglos XVI al XVIII", Analecta Malacitana Electrónica, 25, 2008, 288 p.

García Aranda María de los Ángeles, Un capitulo de la lexicografía didáctica del español: nomenclaturas hispanolatinas (1493-1745), Tesis doctoral de la Universidad Complutense, 2003, 821 p.

Maux-Piovano Marie-Hélène, Les débuts de la didactique de l'espagnol en France : les premières grammaires pratiques (1596-1660), Villeneuve-d'Ascq, Presses Universitaires de Septentrion, 2002, 430 p.

Nieto Jiménez Lidio y Alvar Ezquerra Manuel, Nuevo Tesoro Lexicográfico del Español (siglo XIV-1729), Madrid, Arco/Libros, 2007.

Pablo Núñez Luis, El arte de las palabras: diccionarios e imprenta en el Siglo de Oro, Mérida, Editora Regional de Extremadura, 2010, 465 p.

Quemada Bernard, Les dictionnaires du français moderne 1539-1863: Étude sur leur histoire, leurs types et leurs méthodes, París, Didier, 1968, 684 p. 
Sánchez Regueira Isolina, "César et Antoine Oudin: étude comparative des éditions de son "Thrésor" et son importance dans le domaine de la lexicographie», Verba, 9, 1982, p. 329-340.

— «Antoine Oudin, gramático francés entre Maupas y Vaugelas», Verba, 12, 1985 , p. 361-382.

Suso López Javier, "La enseñanza del español en Francia en el siglo XVII», en González Carrillo Manuel (coord.), Post tenebras spero lucem. Los estudios gramaticales en la España medieval y renacentista, Antonio, Granada, Editorial de la Universidad de Granada, 2010, 538 p. 